\title{
A case report of exceptional clinical response to chemoradiotherapy and tyrosine kinase inhibitors in a patient with EML4-ALK fusion variant 1 non-small cell lung cancer
}

\author{
Xinyan Xu ${ }^{1,2 \#}$, Di Liu ${ }^{1,2 \#}$, Junmiao Wen ${ }^{1,2}$, Jiayan Chen $^{1,2}$, Min Fan $^{1,2}$ \\ ${ }^{1}$ Department of Radiation Oncology, Fudan University Shanghai Cancer Center, Shanghai, China; ${ }^{2}$ Department of Oncology, Shanghai Medical \\ College, Fudan University, Shanghai, China \\ \#These authors contributed equally to this work. \\ Correspondence to: Jiayan Chen, MD; Min Fan, MD, PhD. Department of Radiation Oncology, Fudan University Shanghai Cancer Center, Shanghai \\ 200032, China. Email: chenjiayan2008@126.com; fanmin_fuscc@aliyun.com.
}

\begin{abstract}
Echinoderm microtubule-associated protein-like 4 (EML4)-anaplastic lymphoma kinase (ALK) fusion occurs in approximately $5 \%$ of non-small cell lung cancer (NSCLC) cases. Variants 1 and $3 \mathrm{a} / \mathrm{b}$ are the most common EML4-ALK variants. Emerging evidence indicates that patients with variant 1 and those with variant $3 \mathrm{a} / \mathrm{b}$ exhibit differential therapeutic responses. However, the National Comprehensive Cancer Network guidelines have not included the EML4-ALK fusion subtype in treatment decision-making to date. Herein, we report the case of a non-smoking 36-year-old female patient who was diagnosed with right lung adenocarcinoma in 2005 (cT1N3M0, IIIB) and received definitive chemoradiotherapy. The patient achieved a partial response, and her disease remained under control for 8 years. However, in May 2013, the patient was diagnosed with brain metastasis and underwent subsequent surgical resection, followed by postoperative brain radiotherapy and chemotherapy. Postoperative pathology confirmed ALK gene rearrangement, and next-generation sequencing performed in 2020 identified the EML4-ALK variant as variant 1 . After progression-free survival lasting 4 years, new metastatic lesions were found in the patient's right lung, and she was administered crizotinib for 20 months. Due to a suspicious recurrence in the intracranial surgical margin area, as well as an unbearable gastrointestinal reaction to crizotinib, alectinib was later adopted. At the 7-month follow-up, positron emission tomography/computed tomography revealed a clinical complete response. This case of an NSCLC patient with EML4-ALK fusion variant 1 who exhibited an exceptional response to chemoradiotherapy and ALK inhibitors might broaden horizons in efforts to reveal the molecular mechanism of radiosensitivity in ALK-positive NSCLC and provide reference for further research regarding the optimal radiotherapy delivery dose and tyrosine kinase inhibitor selection.
\end{abstract}

Keywords: Lung cancer; echinoderm microtubule-associated protein-like 4-anaplastic lymphoma kinase (EML4ALK); variant 1; case report

Submitted Oct 21, 2020. Accepted for publication Dec 04, 2020.

doi: $10.21037 /$ tlcr-20-1212

View this article at: http://dx.doi.org/10.21037/tlcr-20-1212

\section{Introduction}

Lung cancer is the leading cause of cancer-related death globally, and non-small cell lung cancer (NSCLC) accounts for more than $80 \%$ of lung cancer cases (1). Approximately $65 \%$ of NSCLC cases are initially diagnosed as locally advanced (stage IIIB) or advanced (stage IV) (2). Anaplastic lymphoma kinase $(A L K)$ gene rearrangements occur in 3-7\% of NSCLC. ALK rearrangements are more common in younger patients with a history of never or light smoking $(3,4)$, and are associated with a high incidence of brain metastases (BM) (5). Specific molecular alterations contribute to tumor heterogeneity in locally advanced or 


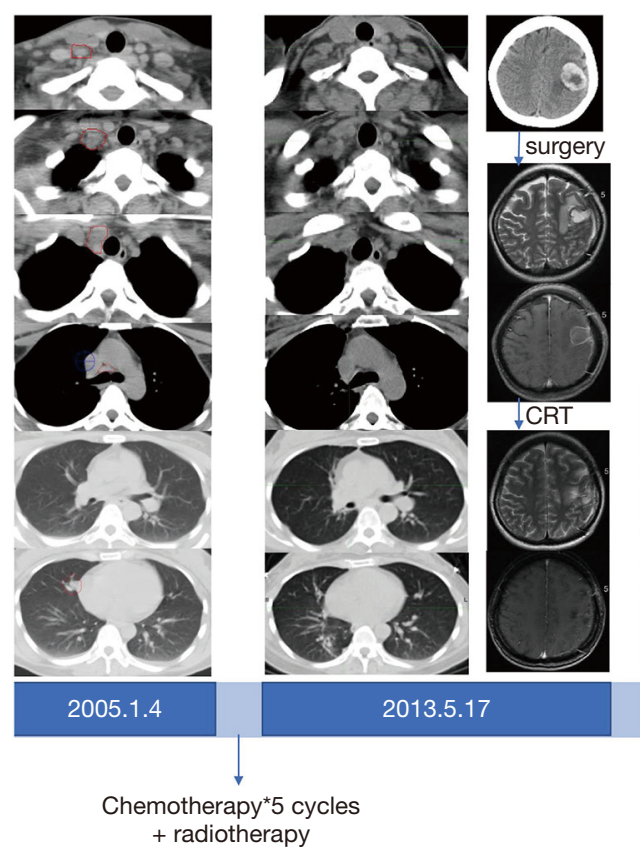

Figure 1 Diagnosis and treatment procedure.
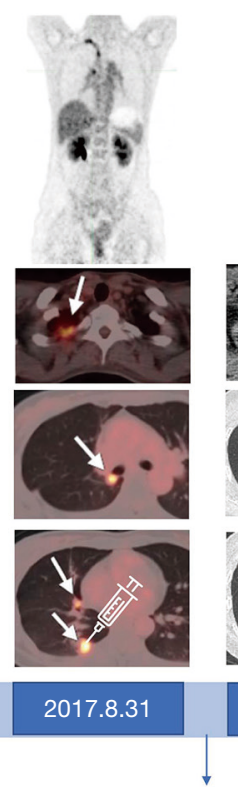

Chemotherapy ${ }^{*} 1$ cycle

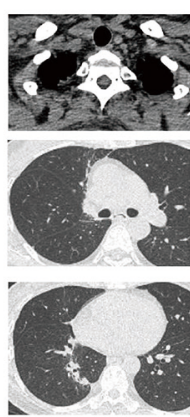

2018.3.13

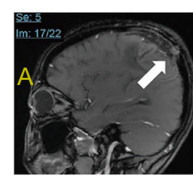

2019.6 .23

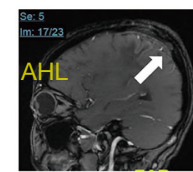

2019.12 .6
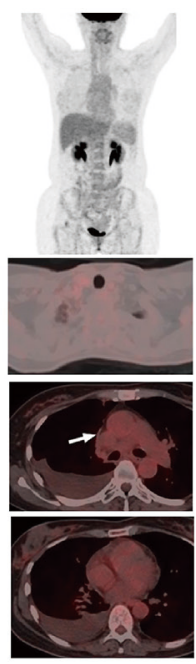

2020.7 .2

advanced NSCLC, which results in variation in treatment efficacy. Herein, we present the case of a patient with ALK-positive variant 1 NSCLC who exhibited a favorable response to treatment with chemoradiotherapy and ALK inhibitors, indicating the inherent therapeutic sensitivity of this specific subtype of lung cancer. We present the following article in accordance with the CARE reporting checklist (available at http://dx.doi.org/10.21037/tlcr-201212).

\section{Case presentation}

A non-smoking 36-year-old female patient was diagnosed with lung cancer originating in the middle and lower lobes of the right lung in January 2005. The patient was staged as cT1N3M0 (overall stage IIIB) according to 6th Edition of American Joint Committee on Cancer (AJCC) TNM Staging System (2002), which was routinely applied in tumor staging at that time (Figure 1). Pathological examination of the right-sided supraclavicular nodes by percutaneous needle biopsy revealed a poorly differentiated adenocarcinoma. Subsequently, the patient underwent sequential definitive chemoradiotherapy. Chemotherapy was administered every 3 weeks for 5 cycles using vinorelbine $\left(25 \mathrm{mg} / \mathrm{m}^{2}\right.$, days 1 and 8$)$ with cisplatin (30 $\mathrm{mg} / \mathrm{m}^{2}$, days $\left.1-3\right)$. A linear accelerator with $6 \mathrm{MV}$ photons was used to deliver a total dose of $6527 \mathrm{cGy}$ [actual mean dose of planning target volume (PTV)] in 31 fractions, with a conventional fractional dose of 2 Gy a day and 5 days a week, to a prescribed isodose of $95 \%$, utilizing a seven-field intensity-modulated radiation therapy (IMRT) technique. The gross tumor volume (GTV) was defined as the primary tumor lesion plus the involved lymph nodes $(1 \mathrm{R}, 2 \mathrm{R}, 4 \mathrm{R})$. The clinical target volume (CTV) was defined as the GTV plus the mediastinal lymphatic drainage area. The PTV was defined as the CTV plus a $1-\mathrm{cm}$ expansion (Figure 2A). The dose-volume histogram (DVH) showed that doses for organs at risk (OARs) were acceptable (Figure 2B). After completing chemoradiotherapy, the patient was observed to have achieved a partial response, and her disease remained under control for 8 years, during which time she was regularly followed up (Figure 1).

In May 2013, the patient began to experience headache and a decrease in grip strength of the right upper limb. Positron emission tomography/computed tomography (PET/CT) was performed and revealed a metastatic lesion, measuring $3.7 \times 2.9 \mathrm{~cm}$, in the left frontal/parietal lobe with a maximum standardized uptake value $\left(\mathrm{SUV}_{\text {max }}\right)$ of 12.6 (Figure 1). She underwent surgical resection, and postoperative pathology confirmed metastatic lung adenocarcinoma (Figure 2C). Fluorescence in situ hybridization (FISH) detected ALK gene rearrangement, 


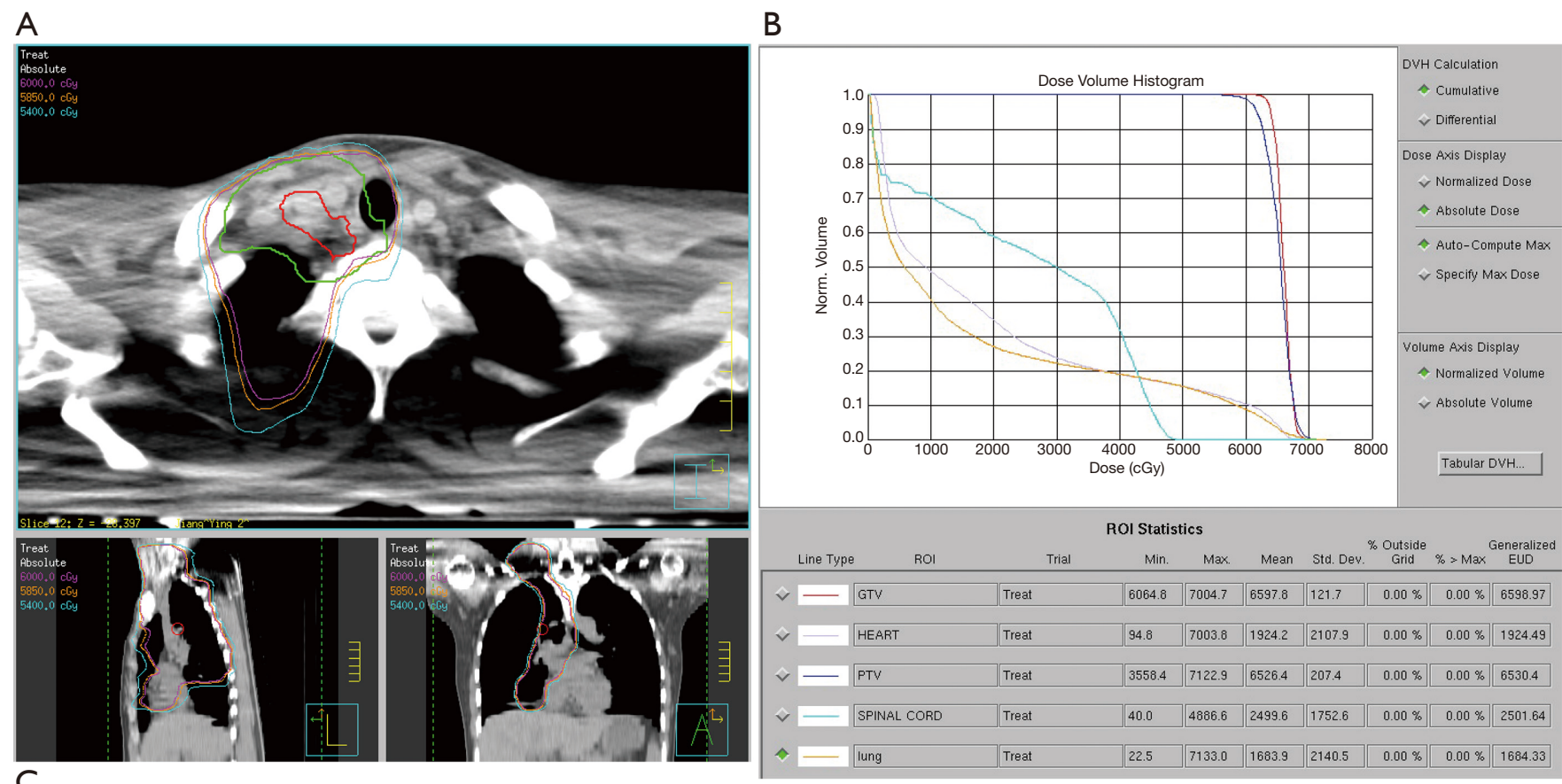

C

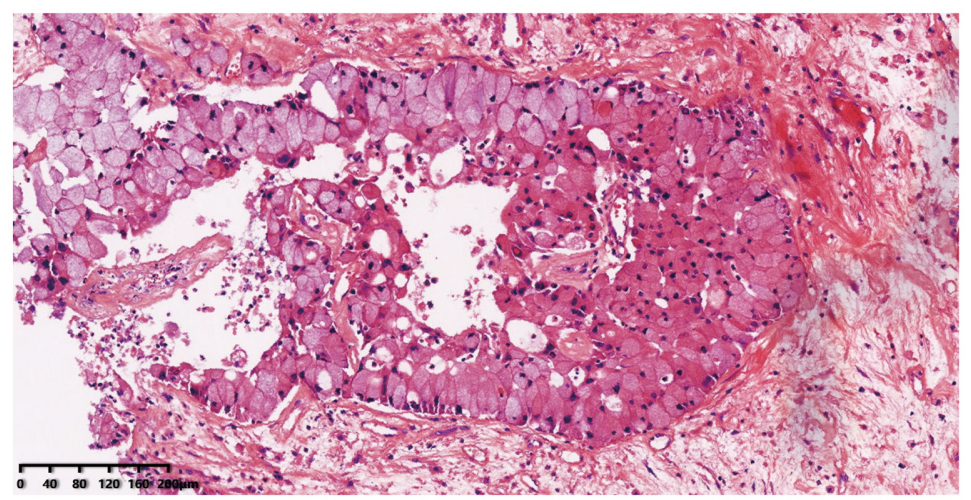

D
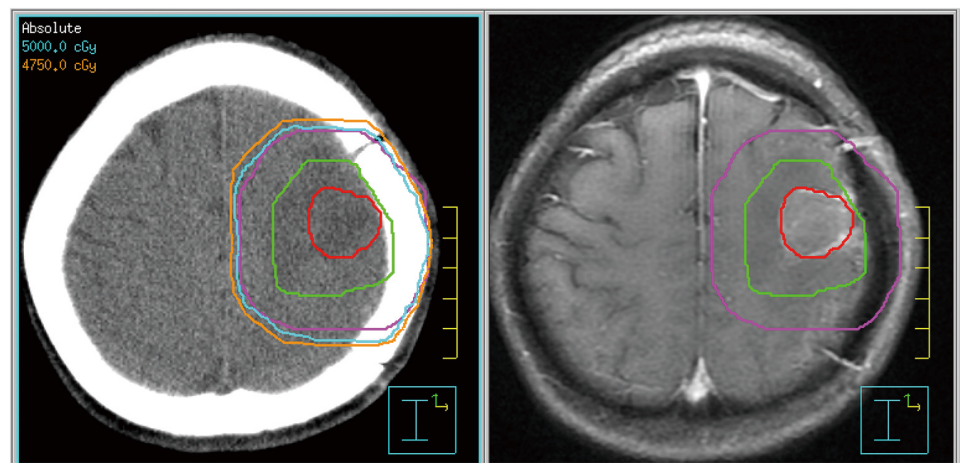

$\mathrm{E}$

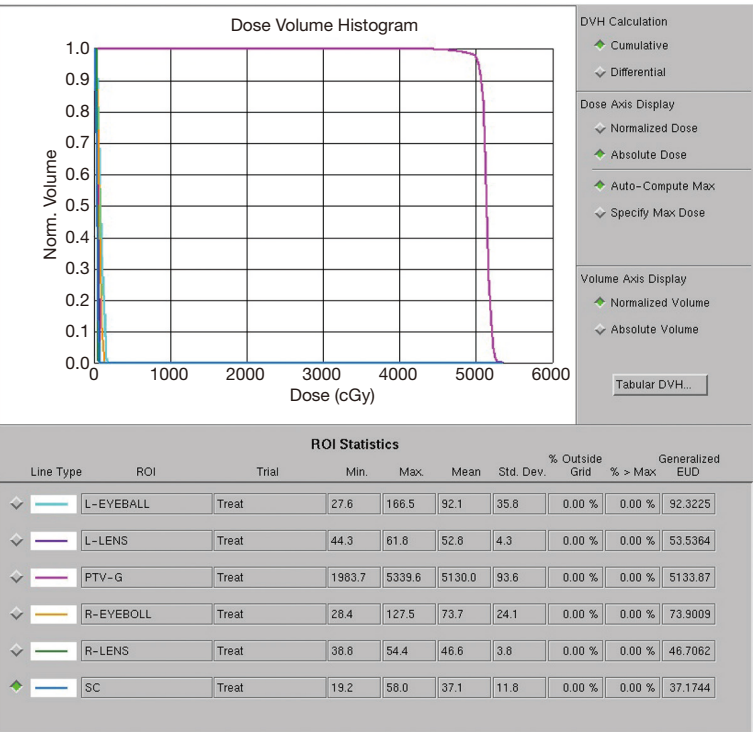

Figure 2 Thoracic and intracranial radiotherapy plans. (A) Contouring of lesions and isodose curves in thoracic radiotherapy. (B) Dosevolume histogram (DVH) of the thoracic radiotherapy plan. (C) Hematoxylin and eosin (HE) staining of a surgically removed intracranial lesion, original magnification $\times 100$. (D) Postoperative radiotherapy plan for brain metastasis. (E) DVH of the intracranial radiotherapy plan. 


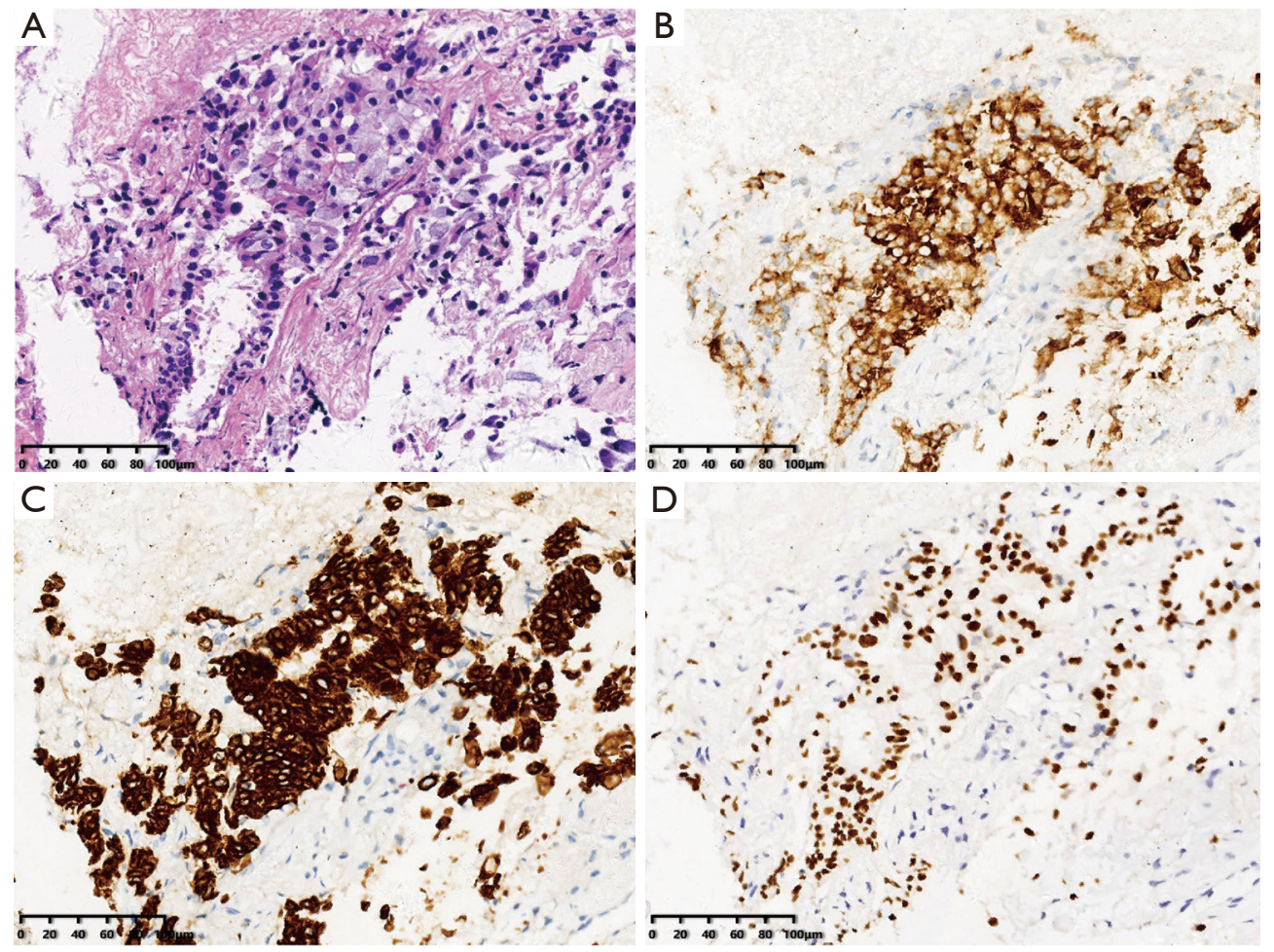

Figure 3 Pathologic findings of a thoracic metastatic biopsy lesion. (A) HE staining, original magnification $\times 200$. (B) ALK rearrangement detected by immunohistochemistry (IHC), original magnification $\times 200$. (C) Cell keratin 7 (CK7) expression detected by IHC, original magnification $\times 200$. (D) Expression of thyroid transcription factor (TTF)-1 by IHC, original magnification $\times 200$.

while exon sequencing revealed no epidermal growth factor receptor mutation. The ALK variant was identified by FoundationOne next-generation sequencing (NGS) assays as variant 1 (V1) in post-hoc analysis (2020). Postoperative brain radiotherapy and chemotherapy was delivered in consideration of intracranial oligometastasis in this patient. For the radiotherapy, the GTV was defined as the tumor bed, the CTV was defined as the tumor bed plus the surrounding edema area, and the PTV was defined as the CTV plus a $1-\mathrm{cm}$ expansion. A dose of 50 Gy was administered in 25 fractions (Figure $2 D, E$ ) and was accompanied by 4 cycles of pemetrexed $\left(500 \mathrm{mg} / \mathrm{m}^{2}\right.$, day 1) combined with cisplatin $\left(75 \mathrm{mg} / \mathrm{m}^{2}\right.$, days $\left.1-3\right)$ every 3 weeks. After treatment, a marked improvement was observed in the patient's primary intracranial symptoms, which was supported by no obvious recurrence or metastasis in brain magnetic resonance (MR) at regular follow-up examinations. However, 4 years later, in August 2017, the patient experienced anhelation and progressive emaciation, and PET/CT discovered new metastatic lesions in the right lung and right pleura, although no significant intracranial recurrence or metastasis was observed. CT-guided needle aspiration of the right pleural lesion confirmed metastatic lung adenocarcinoma (Figures 1,3). One cycle of chemotherapy of pemetrexed $\left(500 \mathrm{mg} / \mathrm{m}^{2}, \mathrm{~d} 1\right)$ combined with carboplatin (AUC $=5$, day 1 ) was delivered, and the patient achieved a partial response.

From March 2018, the patient was prescribed crizotinib, starting with the standard dose of $250 \mathrm{mg}$ bid. However, in response to this treatment, she developed unbearable stomachache and gastric distention accompanied by fatigue, resulting in a dose reduction to $250 \mathrm{mg}$ qd. During treatment with crizotinib, the patient's thoracic disease remained stable, except for a suspicious recurrence in the surgical margin area at the 15-month follow-up (Figure 1). Intracranial disease progression was detected by magnetic resonance imaging at another hospital 5 months later, and considering the patient's persistent gastrointestinal side effects, the administration of crizotinib was discontinued. Since November 2019, the patient has been receiving the second-generation tyrosine kinase inhibitor (TKI) alectinib at dose of $600 \mathrm{mg}$ bid. No adverse events of grade 3 or 
above have occurred so far. It is worth noting that the strengthening signal of the potential intracranial recurrent lesion was weakened after 1 month of treatment with alectinib and has remained stable since then. At the latest follow-up in July, 2020, PET/CT revealed no abnormal hypermetabolic region, indicating a clinical complete response (Figure 1).

Written informed consent was obtained from the patient for publication of this study and any accompanying images. All procedures performed in studies involving human participants were in accordance with the ethical standards of the institutional and/or national research committee(s) and with the Helsinki Declaration (as revised in 2013).

\section{Discussion}

Radical chemoradiotherapy has long been the cornerstone treatment for unresectable stage III NSCLC $(6,7)$, with $\geq 60$ Gy thoracic radiotherapy (8) and two to four cycles of cisplatin-based chemotherapy as the recommended regimen $(9,10)$. The latest National Comprehensive Cancer Network (NCCN) guidelines recommend durvalumab as consolidation therapy only for patients with no disease progression after two or more cycles of definitive chemoradiotherapy (11). However, the general therapeutic effect for unresectable stage III NSCLC patients who receive chemoradiotherapy is unsatisfactory, with median overall survival (OS) of less than 30 months, median progression-free survival (PFS) of less than 12 months, and a 5 -year overall survival rate of less than $30 \%$ (6-10). In the case described above, a patient, who was initially diagnosed as stage IIIB in 2005 and achieved PFS lasting 8 years, benefitted immensely from treatment with definitive chemoradiotherapy, achieving an exceptional therapeutic response. For patients who develop symptomatic intracranial oligometastasis during follow-up, especially those with a large tumor burden, surgical resection followed by stereotactic radiosurgery or whole-brain radiotherapy is deemed an appropriate option by the NCCN guidelines (11). Combined data from the PROFILE 1005 and 1007 trials indicated that brain radiotherapy before crizotinib could prolong the intracranial time to progression from 7.0 to 13.2 months (12). Mak et al. (13) found that the receipt of targeted therapy after cranial irradiation was strongly connected with improved survival. Ni et al. (14) reported that upfront brain radiotherapy followed by crizotinib provided considerable clinical benefits for patients with baseline oligometastatic intracranial lesions.
The treatment strategy for our patient after her diagnosis of intracranial oligometastasis was consistent with those in other studies and achieved moderately better PFS than that previously reported (12).

Specific oncogenic driver alterations may explain the favorable radiotherapeutic response in this case. The most frequent ALK gene translocation partner is echinoderm microtubule-associated protein-like 4 (EML4) (15). Among all variants, the most common are V1 (EML4 exon 13 fused to ALK exon 20, E13;A20) and V3a/b (EML4 exon 6a/b fused to ALK exon 20, E6a/b;A20) (15). Current evidence focusing on radiotherapeutic response in ALKpositive patients is mainly based on cranial radiotherapy in patients with BM. Johung et al. (15) and Arrieta et al. (16) demonstrated that in NSCLC patients with BM, those harboring EGFR mutations and ALK rearrangements have superior radiotherapeutic control rates compared to those with KARS mutations and wild-type (WT) NSCLC. Arrieta et al. also reported that ALK-positive NSCLC patients had longer intracranial PFS than those with WT NSCLC (18.4 vs. 8.7 months), which was comparable to that of patients with EGFR mutation (18.2 months) (16). Mak et al. (13) found that after adjustment for receipt of systemic therapy (TKI and chemotherapy) and baseline characteristics (including number of $\mathrm{BM}$ and presence of extracranial metastases) in patients with BM, those with ALK rearrangements displayed improved survival compared to those with mutations in EGFR or KRAS, or a WT genetic profile. The observations reported in our case are in line with those of previous studies demonstrating the promising response of ALK-positive NSCLC patients to intracranial radiotherapy in terms of long PFS (4 years in this case). To our knowledge, this is the first report of a case of EML4-ALK V1 NSCLC demonstrating an excellent response to primary thoracic radiotherapy, which was reflected in prolonged PFS of 8 years after definitive thoracic chemoradiotherapy. Of note, previous studies on the impact of ALK rearrangements on radiotherapeutic response were mostly based on small patient cohorts, and differential radiosensitivity among ALK variant subtypes has rarely been investigated. Currently, genetic profiles are not taken into consideration in radiotherapy-related decision-making $(11,17)$. This case, in which a patient with EML4-ALK fusion V1 exhibited a favorable response to radiotherapy, could potentially serve as a reference for future dose optimization research for such patients.

Crizotinib is a first-generation TKI that targets ALK gene rearrangements and presents significant benefits in 
regard to survival and intracranial disease control $(3,18)$. The brain remains the most common site of progression in patients with or without BM at initial diagnosis(12). In this case, the patient also experienced suspicious intracranial progression after 15 months of treatment with crizotinib. Alectinib, a second-generation ALK inhibitor that has demonstrated effective central nervous system (CNS) penetration, was notably reported to delay CNS progression versus crizotinib, irrespective of prior CNS disease or brain radiotherapy (19). Mok et al. observed the OS benefit of alectinib in patients with [HR 0.58 (95\% CI, $0.34-1.00)$ ] and without [HR 0.76 (95\% CI, 0.45-1.26)] baseline CNS metastases (20). Of note, alectinib is now recommended as the preferred first-line strategy (11) for patients with ALK-positive advanced NSCLC, based on its outstanding performance in the ALEX study, in which it achieved a median PFS of 34.8 months in untreated ALKpositive patients with advanced NSCLC (20). Moreover, the incidence of adverse events, including gastrointestinal adverse events, was reported to be markedly lower with alectinib than with crizotinib $(21,22)$. In the case of our patient, alectinib considerably relieved severe gastrointestinal side effects associated with crizotinib, and complete response was obtained after 7 months of alectinib treatment. Nonetheless, second-generation TKIs are associated with a higher incidence of developing resistant mutations than first-generation TKIs (23), which diminishes therapeutic options after the failure of second-generation TKI therapy. Hence, investigation of genetic alteration subtypes is critical to deepening our understanding of therapeutic efficacy and improving alternative treatment options.

Patients with $\mathrm{V} 1$ have been reported to achieve longer PFS in response to crizotinib than non-V1 patients (24), while non-V3 patients display a longer PFS than those with V3 (25). The patient in the case described above obtained a PFS of at least 15 months after first-line treatment with crizotinib, even with a dose reduction due to side events. This PFS is superior to the average median PFS of 10.9 months $(3,26)$, and is consistent with the results of a previous study in which patients with V1 achieved better efficacy of crizotinib than non-V1 (24). Lin et al. (23) reported that patients with V3 were more likely to harbor resistance mutations than those with $\mathrm{V} 1$ ( $57 \%$ vs. $30 \%, \mathrm{P}=0.023)$. In particular, they found that those with $\mathrm{V} 3$ were more prone than those with V1 to develop G1202R mutation (32\% vs. $0 \%, \mathrm{P}<0.001$ ), which is more common in patients resistant to second-generation TKIs and only showed a response to the third-generation TKI lorlatinib (27). It has been indicated that among patients receiving second-generation ALK inhibitors after crizotinib, those with V1 have a longer PFS than those with V3, which to some degree explains the excellent performance of alectinib in the case of our patient. However, with the administration of lorlatinib after firstand second-generation TKI therapy, patients with V3 tended to outperform those with V1 in terms of PFS (23). Although prospective phase III data from the ALEX study (26) did not show a significant difference in therapeutic efficacy between $\mathrm{V} 1$ and $\mathrm{V} 3 \mathrm{a} / \mathrm{b}$ with crizotinib or alectinib in the first-line setting, there is still potential to incorporate ALK variants into efficacy prediction and TKI treatment decision-making. To the best of our knowledge, the ALK rearrangement subtype of V1 may have contributed to the favorable response to TKIs in this case, leaving next-step treatment alternatives to be discussed when this patient eventually acquires resistance to alectinib.

\section{Conclusions}

Here, we have presented the case of a NSCLC patient with EML4-ALK fusion V1 who exhibited a favorable response to chemoradiotherapy as well as ALK inhibitors. Variant subtypes of EML4-ALK may be potential research targets for further studies regarding differentiated responses to radiotherapy in such patients, leaving deepened mechanism researches to be conducted in the future. Up to now, the NCCN guidelines have not included the EML4-ALK fusion subtype into treatment decision-making, neither in relation to radiotherapy nor TKIs (11). This case might broaden horizons in uncovering the differentiated radiosensitivity of NSCLC with different EML4-ALK variants, paving the way for further retrospective and prospective studies regarding optimized radiotherapy delivery dose and TKI selection in the future.

\section{Acknowledgments}

Funding: Clinical Research Plan of SHDC (SHDC2020CR3025B), CSCO-Leading Cancer Research Fund (Y-2019AZZD-0561), CSCO-MSD Cancer Research Fund (Y-MSD2020-0336), Lian Yun Gang Shi Hui Lan Public Foundation (HL-HS2020-66).

\section{Footnote}

Reporting Checklist: The authors have completed the CARE reporting checklist. Available at http://dx.doi.org/10.21037/ 
tlcr-20-1212

Conflicts of Interest: All authors have completed the ICMJE uniform disclosure form (available at http://dx.doi. org/10.21037/tlcr-20-1212). The authors have no conflicts of interest to declare.

Ethical Statement: The authors are accountable for all aspects of the work in ensuring that questions related to the accuracy or integrity of any part of the work are appropriately investigated and resolved. Written informed consent was obtained from the patient for publication of this study and any accompanying images. All procedures performed in studies involving human participants were in accordance with the ethical standards of the institutional and/or national research committee(s) and with the Helsinki Declaration (as revised in 2013).

Open Access Statement: This is an Open Access article distributed in accordance with the Creative Commons Attribution-NonCommercial-NoDerivs 4.0 International License (CC BY-NC-ND 4.0), which permits the noncommercial replication and distribution of the article with the strict proviso that no changes or edits are made and the original work is properly cited (including links to both the formal publication through the relevant DOI and the license). See: https://creativecommons.org/licenses/by-nc-nd/4.0/.

\section{References}

1. Siegel RL, Miller KD, Jemal A. Cancer statistics, 2018. CA Cancer J Clin 2018;68:7-30.

2. William WN Jr, Lin HY, Lee JJ, et al. Revisiting stage IIIB and IV non-small cell lung cancer: analysis of the surveillance, epidemiology, and end results data. Chest 2009;136:701-9.

3. Solomon BJ, Mok T, Kim DW, et al. First-line crizotinib versus chemotherapy in ALK-positive lung cancer. $\mathrm{N}$ Engl J Med 2014;371:2167-77.

4. Mogenet A, Tomasini P, Greillier L, et al. Lorlatinib: an additional option for ALK-positive non-small cell lung cancer? Transl Lung Cancer Res 2019;8:S383-6.

5. Johung KL, Yeh N, Desai NB, et al. Extended Survival and Prognostic Factors for Patients With ALK-Rearranged Non-Small-Cell Lung Cancer and Brain Metastasis. J Clin Oncol 2016;34:123-9.

6. Curran WJ, Paulus R, Langer CJ, et al. Sequential vs. concurrent chemoradiation for stage III non-small cell lung cancer: randomized phase III trial RTOG 9410. J

Natl Cancer Inst 2011;103:1452-60.

7. Aupérin A, Le Péchoux C, Rolland E, et al. Meta-analysis of concomitant versus sequential radiochemotherapy in locally advanced non-small-cell lung cancer. J Clin Oncol 2010;28:2181-90.

8. Bradley JD, Paulus R, Komaki R, et al. Standarddose versus high-dose conformal radiotherapy with concurrent and consolidation carboplatin plus paclitaxel with or without cetuximab for patients with stage IIIA or IIIB non-small-cell lung cancer (RTOG 0617): a randomised, two-by-two factorial phase 3 study. Lancet Oncol 2015;16:187-99.

9. Senan S, Brade A, Wang LH, et al. PROCLAIM: Randomized Phase III Trial of Pemetrexed-Cisplatin or Etoposide-Cisplatin Plus Thoracic Radiation Therapy Followed by Consolidation Chemotherapy in Locally Advanced Nonsquamous Non-Small-Cell Lung Cancer. J Clin Oncol 2016;34:953-62.

10. Liang J, Bi N, Wu S, et al. Etoposide and cisplatin versus paclitaxel and carboplatin with concurrent thoracic radiotherapy in unresectable stage III non-small cell lung cancer: a multicenter randomized phase III trial. Ann Oncol 2017;28:777-83.

11. National Comprehensive Cancer Network. NCCN Clinical Practice Guidelines in Oncology: Non-Small Cell Lung Cancer Version 3.2020. Available online: https://www.nccn.org/professionals/physician_gls/default. aspx\#nscl. Accessed August 12, 2020.

12. Costa DB, Shaw AT, Ou SH, et al. Clinical Experience With Crizotinib in Patients With Advanced ALKRearranged Non-Small-Cell Lung Cancer and Brain Metastases. J Clin Oncol 2015;33:1881-8.

13. Mak KS, Gainor JF, Niemierko A, et al. Significance of targeted therapy and genetic alterations in EGFR, ALK, or KRAS on survival in patients with non-small cell lung cancer treated with radiotherapy for brain metastases. Neuro Oncol 2015;17:296-302.

14. Ni J, Li G, Yang X, et al. Optimal timing and clinical value of radiotherapy in advanced ALK-rearranged non-small cell lung cancer with or without baseline brain metastases: implications from pattern of failure analyses. Radiat Oncol 2019;14:44.

15. Johung KL, Yao X, Li F, et al. A clinical model for identifying radiosensitive tumor genotypes in non-small cell lung cancer. Clin Cancer Res 2013;19:5523-32.

16. Arrieta O, Ramirez-Tirado LA, Caballe-Perez E, et al. Response rate of patients with baseline brain metastases 
from recently diagnosed non-small cell lung cancer receiving radiotherapy according to EGFR, ALK and KRAS mutation status. Thorac Cancer 2020;11:1026-37.

17. Cassidy RJ, Zhang X, Patel PR, et al. Next-generation sequencing and clinical outcomes of patients with lung adenocarcinoma treated with stereotactic body radiotherapy. Cancer 2017;123:3681-90

18. Solomon BJ, Cappuzzo F, Felip E, et al. Intracranial Efficacy of Crizotinib Versus Chemotherapy in Patients With Advanced ALK-Positive Non-Small-Cell Lung Cancer: Results From PROFILE 1014. J Clin Oncol 2016;34:2858-65.

19. Gadgeel S, Peters S, Mok T, et al. Alectinib versus crizotinib in treatment-naive anaplastic lymphoma kinase-positive (ALK+) non-small-cell lung cancer: CNS efficacy results from the ALEX study. Ann Oncol 2018;29:2214-22.

20. Mok T, Camidge DR, Gadgeel SM, et al. Updated overall survival and final progression-free survival data for patients with treatment-naive advanced ALK-positive non-small-cell lung cancer in the ALEX study. Ann Oncol 2020;31:1056-64.

21. Cho BC, Obermannova R, Bearz A, et al. Efficacy and Safety of Ceritinib $(450 \mathrm{mg} / \mathrm{d}$ or $600 \mathrm{mg} / \mathrm{d})$ With Food Versus $750-\mathrm{mg} / \mathrm{d}$ Fasted in Patients With ALK Receptor Tyrosine Kinase (ALK)-Positive NSCLC: Primary Efficacy Results From the ASCEND-8 Study. J Thorac Oncol 2019;14:1255-65.

Cite this article as: $\mathrm{Xu} \mathrm{X}$, Liu D, Wen J, Chen J, Fan M. A case report of exceptional clinical response to chemoradiotherapy and tyrosine kinase inhibitors in a patient with EML4-ALK fusion variant 1 non-small cell lung cancer. Transl Lung Cancer Res 2020;9(6):2500-2507. doi: 10.21037/tlcr-20-1212
22. Peters S, Camidge DR, Shaw AT, et al. Alectinib versus Crizotinib in Untreated ALK-Positive Non-Small-Cell Lung Cancer. N Engl J Med 2017;377:829-38.

23. Lin JJ, Zhu VW, Yoda S, et al. Impact of EML4ALK Variant on Resistance Mechanisms and Clinical Outcomes in ALK-Positive Lung Cancer. J Clin Oncol 2018;36:1199-06.

24. Yoshida T, Oya Y, Tanaka K, et al. Differential Crizotinib Response Duration Among ALK Fusion Variants in ALKPositive Non-Small-Cell Lung Cancer. J Clin Oncol 2016;34:3383-9.

25. Woo CG, Seo S, Kim SW, et al: Differential Protein Stability and Clinical Responses of EML4-ALK Fusion Variants to Various ALK Inhibitors in Advanced ALKrearranged Non-small Cell Lung Cancer. Ann Oncol 2017;28:791-7.

26. Camidge DR, Dziadziuszko R, Peters S, et al. Updated Efficacy and Safety Data and Impact of the EML4-ALK Fusion Variant on the Efficacy of Alectinib in Untreated ALK-Positive Advanced Non-Small Cell Lung Cancer in the Global Phase III ALEX Study. J Thorac Oncol 2019;14:1233-43.

27. Gainor JF, Dardaei L, Yoda S, et al. Molecular Mechanisms of Resistance to First- and Second-Generation ALK Inhibitors in ALK-Rearranged Lung Cancer. Cancer Discov 2016;6:1118-33.

(English Language Editor: J. Reynolds) 